\title{
Glycosidic aroma compounds of some Portuguese grape cultivars
}

\author{
Maria João Cabrita, ${ }^{1 *}$ Ana M Costa Freitas, ${ }^{1}$ Olga Laureano ${ }^{2}$ and Rocco Di Stefano ${ }^{3}$ \\ ${ }^{1}$ Departamento de Fitotecnia, Instituto de Ciências Agrárias e Mediterrânicas-ICAM, Universidade de Évora, Apt 94 7002-554 Évora, \\ Portugal \\ ${ }^{2}$ Laboratório Ferreira Lapa (Sector de Enologia), Instituto Superior de Agronomia, Universidade Técnica de Lisboa, $1349-017$ Lisboa, \\ Portugal \\ ${ }^{3}$ Istituto Sperimental per L'Enologia Asti, Via Pietro Micca 3514100 Asti, Italy
}

\begin{abstract}
In order to evaluate the potential aroma of some Portuguese grapes, several glycosidically bound aroma compounds of ten grape varieties grown in Évora, Alentejo, Portugal, were released, identified and quantified using gas chromatography-mass spectrophotometry. White varieties were Arinto, Perrum, Rabo-de-Ovelha, Roupeiro and Antão Vaz. Red varieties were Trincadeira, Aragonez, Tinta Caiada, Moreto and Castelão. Grapes of Roupeiro and Trincadeira from five other vines in the Alentejo region were also studied. For each variety pulp and skin were analyzed separately. The compounds produced by enzymatic hydrolysis of glycoside precursors extracted from pulps and skins of these grapes were similar to the ones appearing in the so-called 'aromatic' varieties; however, the amounts found were significantly lower. These results seem to show the neutral characteristic of these grapes to indicate that each variety has a distinctive profile regarding compounds from the linalool class. The ratios of trans: cis forms of some linalool compounds remain the same in all samples of Roupeiro and Trincadeira, regardless their origin. In Trincadeira grapes no linalool was detected. Skins are always richer than pulps. The acid hydrolysis of norisoprenoid aglycons produced highly odorant compounds previously described in grape varieties. Although some differences between varieties could be detected, the red grapes Aragonez and Castelão are richer in vitispiranes while Moreto, Trincadeira and Tinta Caiada are richer in actinidols; in white grapes, only Roupeiro and Perrum show detectable amounts of vitispiranes. These results need further investigation and analysis to be considered as a statement and should be confirmed in an extended study.
\end{abstract}

(C) 2006 Society of Chemical Industry

Keywords: aroma; glycosides; Portuguese grape cultivars

\section{INTRODUCTION}

All cultivars of Vitis vinifera studied up until now show differences in the composition of secondary metabolites such as terpene alcohols, norisoprenoids and benzenoids sufficiently great to justify their use in varietal characterization. ${ }^{1}$

The typical flavour of wines is mainly due to volatile compounds that proceed from the grapes where they are in free volatile form or in bound form, usually as glycosides. In most cases these glycosides accumulate in the fruit and can be found in higher amounts than in the free forms. ${ }^{2}$

Chemical analyses of volatile secondary metabolites of wine grapes are increasingly employed to understand the influence of grape variety on wine flavour. While volatile metabolites are found in low concentrations in grapes, they usually accumulate in much higher concentrations as bound forms, mainly as flavourless glycoconjugates, which can be an additional reservoir of flavour, releasing further volatile compounds via mild acid hydrolysis that takes place naturally during vinification and storage of wines. ${ }^{3}$ The analysis of volatiles released from flavourless glycoconjugates is a valuable strategy for flavour studies on fruits that contain low concentrations of free aroma compounds. ${ }^{4}$

According to the quantities of free and bound forms of varietal compounds, grapes can be classified as neutral or aromatic, the last having higher levels of terpenic compounds. Neutral grapes also have terpenic compounds and, like the aromatic ones, we can split them into classes of the same biosynthetic activities. Aroma compounds identified in grapes have been found to differ in quantity with the variety and to pass more or less unchanged into the wine aroma, thus being considered for variety classification and/or characterization. The growing area only marginally influences the characteristic varietal composition of the monoterpene compounds in the various grape varieties. ${ }^{5}$ Agronomic and climatic aspects have greater influence on the amounts rather than in the type of the aroma compounds present in a grape variety.

Terpenic compounds found in grape cultivars may be grouped into classes having structures similar to linalool and geraniol. Cyclic compounds are related to nerol. Compounds of the linalool class include isomers of furan and pyran oxides of

* Correspondence to: Maria João Cabrita, Departamento de Fitotecnia, Instituto de Ciências Agrárias e Mediterrânicas-ICAM, Universidade de Évora, Apt 94 7002-554 Évora, Portugal

E-mail: mjbc@uevora.pt

Contract/grant sponsor: PAMAF 6007

(Received 12 February 2005; revised version received 27 June 2005; accepted 20 October 2005)

Published online 20 March 2006; DOI: 10.1002/jsfa.2439 
linalool, $E$-3,7-dimethyl-3,7-dihydroxi-1,5-octadiene 3,7-dimethyl-3,7-dihydroxi-1-octene, and $E$ - and $Z$ -

2,6-dimethyl-2,7-octadien-1,6-diol, while compounds of the geraniol class include geraniol, citronellol, geranic acid, 3,7-dimethyl-1,7-octadiol, and Z-3,7-dimethyl-2-en-1,7-octandiol E-3,7-dimethyl-2en-1,7-octandiol. Nerol, $\alpha$-terpineol, $Z$-3,7-dimethyl2-en-1,7-octandiol and $p$-menthene-7,8-diol may be grouped into a nerol class. ${ }^{1}$ All these compounds are present in grapes in free and glycosidically conjugated forms. ${ }^{6,7}$ The bound fraction of the terpenic compounds is usually higher than the free fraction, ${ }^{8}$ even though the glycosidically conjugated forms of the monoterpenes make no direct contribution to the aroma of grapes. $^{9}$

Although the origin of norisoprenoids is yet to be clarified, recent results ${ }^{10}$ support the hypothesis that $\mathrm{C}_{13}$ glycosylated norisoprenoids in grape berries derive exclusively from carotenoid. The relation of the norisoprenoid content to xanthophyll degradation was also considered since xanthophylls decrease during ripening while the norisoprenoid content increases. ${ }^{1} \mathrm{C}_{13}$-Norisoprenoids are considered as those that contribute mostly to the aroma of wines made with non-aromatic grapes.

There are many compounds already identified upon enzymatic hydrolysis of glycoside compounds extracted from berries; most show a megastigmane ring: 3-hydroxymegastigma-5,8-dien7-one, 9-hydroxymegastigma-4,7-dien-3-one and 6,9dihydroxymegastigma-4,7-dien-3-one. By chemical hydrolysis at $\mathrm{pH} 3$ and $100^{\circ} \mathrm{C}$ norisoprenoids produce volatile compounds with a small odour threshold, namely damascenone, Riesling acetal, vitispirane isomers, actinidol isomers and TDN (1,1,6-trimethyl1,2-dihydronaphtalene). ${ }^{11}$

Knowledge of the distribution of glycosidically bound compounds between skins and pulp in grapes used for winemaking offers a valuable guide in applying skin contact, press conditions and pomace contact time to optimize wine aroma. ${ }^{12}$ In fact it is known that although monoterpenes are the compounds responsible for part of the aroma in wine, the so-called PVT (potentially volatile monoterpenes) constitute the major fraction of monoterpenes in wines and as such they are odourless. When released, or if released, however, their aroma thresholds vary from $100-130 \mu \mathrm{gL}^{-1}$ to $400-500 \mu \mathrm{g} \mathrm{mL}^{-1}$ or even $3-5 \mathrm{mg} \mathrm{mL}^{-1}$ depending on the monoterpene considered. Besides the terpene alcohols, known as C13-norisoprenoids, frequently have attractive sensory qualities combined with low flavour thresholds (the lowest concentrations at which they can be smelt) and have been found to be important aroma constituents of wine; thus their release can be beneficial to the final wine aroma. ${ }^{13}$ The beneficial effect of these practices may, however, be overridden by the deleterious effects on wine flavour and colour resulting from the simultaneous extraction of bitter and astringent phenolic compounds, which are located primarily in grape skins.

Studies in varietal characterization have been carried out for some white varieties from the north of Portugal $^{14,15}$ as well as for a few Portuguese red varieties. $^{16,17}$

The present study is a first step in the varietal characterization of the most important grapes of the Alentejo region in the south of Portugal, based on the composition of their glycosidic flavour precursors. Ten varieties - Roupeiro, Arinto, Rabo de Ovelha, Antão Vaz and Perrum for white varieties and Trincadeira, Moreto, Castelão Aragonez and Tinta Caiada for red varieties - all from vines in Évora, were followed during a one-year harvest. Trincadeira and Roupeiro from the other five sub-regions of Alentejo, namely Reguengos, Redondo, Vidigueira, Borba and Portalegre, were also studied in order to evaluate the possible impact of different agronomic and climatic factors on the composition of glycosidic flavour precursors. Because they are usually known as non-floral varieties (neutral) a low concentration of free aroma compounds would be expected. Flavour precursors analysis has been used as a strategy to determine the real flavour potential of these Portuguese varieties, thus giving hints for future technological changes/improvements related to skin and/or pomace contact time as well as the use of enzymes during winemaking to release the bound aroma.

\section{EXPERIMENTAL \\ Grapes}

The grapes used for this work were picked during the 1998 harvest from vines in Évora, Alentejo, Portugal - five white varieties: Roupeiro, Antão Vaz, Perrum, Rabo de Ovelha and Arinto; and five red varieties: Trincadeira, Aragonez, Tinta Caiada, Moreto and Castelão. All these varieties of Vitis vinifera $\mathrm{L}$. are recommended in the Alentejo region, and were picked at commercial maturation. Grapes of Roupeiro and Trincadeira varieties were also picked from vines in Portalegre, Borba, Redondo, Reguengos and Vidigueira, the most important subregions in Alentejo besides Évora. From each variety only one sample (ca $1000 \mathrm{~g}$ ) was collected in all cases; grapes within a sample were randomly picked within the whole vine according to a previously proposed scheme. ${ }^{18}$

\section{Sample preparation}

Aroma compounds were extracted following a previously reported method ${ }^{19,20}$ with modifications. The aroma content in pulps and skins was separately analysed. A sample of 100 berries was peeled. Skins were placed in $20 \mathrm{~mL}$ of methanol and frozen until analysis. Pulps were collected in a beaker, $100 \mathrm{mg}$ 
$\mathrm{Na}_{2} \mathrm{SO}_{3}$ was added and the mixture was frozen until further analysis. After being defrozen, skins were homogenized in an Omni-Mixer (Sorvall, Labequip, Ontario, Canada), centrifuged for $15 \mathrm{~min}$ at $8000 \times \mathrm{g}$; the liquid phase was recovered. The pellet was washed with water and centrifuged again. This last operation was repeated twice. The liquid phases were assembled until $250 \mathrm{~mL}$ of 'skin extract' was obtained. To remove phenolic compounds $1 \mathrm{~g}$ of polyvinylpolypyrrolidone (Sigma, St Louis, MO, USA) was added to the skin extract. After defreezing pulps were also homogenized and centrifuged for $15 \mathrm{~min}$ at $8000 \times \mathrm{g}$. The juice was recovered, while the pellet was washed with water and centrifuged twice. The liquids were assembled until $250 \mathrm{~mL}$ extract was obtained. $100 \mathrm{mg}$ of a commercial pectolitic enzyme (Vinozym, Novo Nordisk Ferment Ltd, Dittingen), without glycosidase activity was added, and after $4 \mathrm{~h}$ centrifuged. $200 \mathrm{~mL}$ of these extracts was eluted through a Sep Pack ${ }^{\circledR}$ Cartridge C18 5 g (Waters, Milford, MA, USA) previously activated with $25 \mathrm{~mL}$ of methanol and $50 \mathrm{~mL}$ of water in sequence. After elution the cartridge was washed with $50 \mathrm{~mL}$ of water followed by $30 \mathrm{~mL}$ of dichloromethane. The bound compounds were eluted with $30 \mathrm{~mL}$ of methanol. Methanol was eliminated under vacuum and the residue solubilized in $5 \mathrm{~mL}$ of a phosphate-citrate buffer $\mathrm{pH} 5$. The glycosidically bound fraction of skins or juice, isolated on

Table 1. Compounds released by enzymatic hydrolysis from the glycosylated precursors extracted from red grapes

\begin{tabular}{|c|c|c|c|c|c|c|c|c|c|c|}
\hline \multirow[b]{2}{*}{ Compounds $\left(\mu \mathrm{g} \mathrm{kg}^{-1}\right)$} & \multicolumn{2}{|c|}{$\mathrm{T}(\mathrm{E})$} & \multicolumn{2}{|c|}{$M(E)$} & \multicolumn{2}{|c|}{$\mathrm{TC}(\mathrm{E})$} & \multicolumn{2}{|c|}{$A z(E)$} & \multicolumn{2}{|c|}{$C(E)$} \\
\hline & Skin & Pulp & Skin & Pulp & Skin & Pulp & Skin & Pulp & Skin & Pulp \\
\hline \multicolumn{11}{|l|}{ C6 compounds } \\
\hline Hexanol & 122.0 & 113.3 & 87.9 & 40.7 & 106.5 & 75.6 & 77.1 & 60.7 & 61.8 & 37.5 \\
\hline trans-3-Hexenol & 6.9 & 9.5 & nd & 2.2 & 12.6 & 2.8 & 1.7 & 2.5 & nd & 1.4 \\
\hline cis-3-Hexenol & 21.6 & 20.2 & 50.4 & 30.3 & 52.1 & 44.7 & 28.0 & 25.6 & 20.9 & 16.8 \\
\hline trans-2-Hexenol & 33.9 & 21.1 & 32.5 & 8.2 & 33.3 & 13.5 & 9.9 & 7.0 & 16.7 & 10.1 \\
\hline Total & 184.4 & 164.1 & 170.8 & 81.4 & 204.5 & 136.6 & 116.7 & 95.8 & 99.4 & 65.8 \\
\hline \multicolumn{11}{|l|}{ Terpenes } \\
\hline trans-Furan linalool oxide & 16.1 & 6.5 & 14.6 & 2.6 & 4.9 & 6.6 & 2.2 & 2.2 & 15.4 & 9.7 \\
\hline cis-Furan linalool oxide & 12.8 & 4.7 & 18.7 & 3.7 & 3.6 & 5.6 & 6.0 & 2.6 & 13.2 & 9.4 \\
\hline trans-Pyran linalool oxide & 33.5 & 9.5 & 23.6 & 7.5 & nd & nd & 3.4 & 3.3 & 12.1 & 8.8 \\
\hline cis-Pyran linalool oxide & 20.2 & 9.5 & 34.2 & 8.2 & nd & nd & 4.3 & 3.3 & 10.9 & 10.9 \\
\hline Nerol & nd & 2.6 & nd & 3.7 & nd & 9.0 & nd & 2.9 & nd & 2.9 \\
\hline Geraniol & 13.3 & 15.9 & 46.4 & 12.0 & 27.9 & 29.4 & 29.3 & 9.5 & 39.2 & 16.3 \\
\hline Z-2,6-Dimethyl-2,7-octadiene-1,6-diol & 14.9 & 14.2 & 38.7 & 12.1 & 7.8 & nd & 10.2 & 7.8 & 28.0 & 7.9 \\
\hline E-2,6-Dimethyl-2,7-octadiene-1,6-diol & 33.2 & 21.7 & 64.2 & 14.6 & 18.0 & 12.4 & 25.2 & 17.5 & 13.2 & 14.1 \\
\hline E-3,7-Dimethyl-2-en-1,7-octandiol & 10.0 & 11.8 & 23.6 & 8.5 & 9.0 & 4.4 & 13.4 & 9.1 & 37.6 & 4.9 \\
\hline Geranic acid & 10.0 & 6.8 & 16.0 & 4.2 & 14.4 & 4.3 & 30.5 & 3.9 & 52.1 & 4.0 \\
\hline p-Menthene-7,8-diol & 10.8 & nd & 21.7 & 23.1 & 72.2 & nd & 6.4 & nd & 9.7 & $\mathrm{Nd}$ \\
\hline Total & 174.8 & 103.2 & 301.7 & 100.2 & 157.8 & 71.7 & 130.9 & 62.1 & 231.4 & 88.9 \\
\hline \multicolumn{11}{|l|}{ Benzenoids } \\
\hline Benzaldehyde & 4.6 & 4.7 & 13.0 & 5.2 & 36.8 & 26.4 & 3.4 & 2.7 & 6.7 & 3.2 \\
\hline Methyl salicylate & 13.3 & 28.8 & 10.6 & 18.7 & 11.7 & 14.9 & 2.6 & 2.2 & 36.7 & 71.3 \\
\hline$\alpha$-Methyl-benzenemethanol & nd & 2.1 & nd & 1.9 & nd & 5.7 & nd & 1.9 & nd & 1.6 \\
\hline Eugenol & nd & 7.4 & 9.4 & 9.1 & 6.0 & 4.2 & 4.8 & 6.7 & 3.5 & nd \\
\hline Benzyl alcohol & 378.8 & 323.3 & 428.4 & 238.0 & 218.4 & 238.8 & 230.0 & 184.9 & 390.2 & 203.0 \\
\hline 2-Phenylethanol & 221.5 & 160.3 & 245.3 & 118.0 & 149.4 & 114.7 & 242.1 & 116.8 & 55.9 & 82.5 \\
\hline 4-Hydroxy-3-methoxyphenethanol & 33.2 & 60.8 & 43.4 & 40.7 & 71.3 & 64.3 & 44.0 & 51.8 & 39.9 & 8.0 \\
\hline Dihydroconiferyl alcohol & 22.4 & 58.0 & 18.9 & 81.4 & 15.0 & 17.4 & 8.0 & 23.7 & 8.4 & 5.9 \\
\hline Total & 673.8 & 645.4 & 769 & 513 & 508.6 & 486.4 & 534.9 & 390.7 & 541.3 & 375.5 \\
\hline \multicolumn{11}{|l|}{ Phenols } \\
\hline 4-Vinylphenol & 16.6 & 9.3 & 18.9 & 75.3 & 114.9 & 33.6 & 100.3 & 68.8 & 222.1 & 28.1 \\
\hline 4-Vinylguaicol & 18.3 & 22.3 & 21.7 & 113.9 & 2.4 & 44.7 & 5.9 & 31.1 & 10.9 & 41.3 \\
\hline Total & 34.9 & 31.6 & 40.6 & 189.2 & 117.3 & 78.3 & 106.2 & 99.9 & 233 & 69.4 \\
\hline \multicolumn{11}{|l|}{ Norisoprenoids } \\
\hline 3-Hydroxy- $\beta$-damascone ${ }^{a}$ & 14.3 & 11.1 & 55.7 & 21.8 & 78.4 & 35.1 & 5.0 & 41.8 & 22.5 & 21.2 \\
\hline $3-\mathrm{O} \times 0-\alpha-$-ionol $^{\mathrm{b}}$ & 18.1 & 23.5 & 81.2 & 27.3 & 69.0 & 29.3 & 46.7 & 71.4 & 35.4 & 7.1 \\
\hline 3,9-Dihydroxy-mega-5-ene & nd & 35.9 & 33.0 & 14.6 & 34.5 & 8.9 & 45.4 & 53.9 & 12.9 & 7.7 \\
\hline Vomifoliol $^{\mathrm{C}}$ & 192.6 & 60.8 & 219.7 & 113.9 & 262.0 & 133.3 & 136.1 & 155.3 & 282.4 & 77.8 \\
\hline Total & 225 & 131.3 & 389.6 & 177.6 & 443.9 & 206.6 & 233.2 & 322.4 & 353.2 & 113.8 \\
\hline
\end{tabular}

T, Trincadeira; M, Moreto; TC, Tinta Caiada; Az, Aragonez; C, Castelão; (E), Évora.

a 3-Hydroxymegastigma-5,8-diene-7-one.

b 9-Hydroxymegastigma-4,7-diene-3-one.

c 6,9-Dihydroxymegastigma-4,7-diene-3-one. 
Table 1A. Compounds released by enzymatic hydrolysis from the glycosylated precursors extracted from red grapes

\begin{tabular}{|c|c|c|c|c|c|c|c|c|c|c|}
\hline \multirow[b]{2}{*}{ Compounds $\left(\mu \mathrm{g} \mathrm{kg}^{-1}\right)$} & \multicolumn{2}{|c|}{$\mathrm{T}(\mathrm{P})$} & \multicolumn{2}{|c|}{$\mathrm{T}(\mathrm{B})$} & \multicolumn{2}{|c|}{$\mathrm{T}(\mathrm{Rd})$} & \multicolumn{2}{|c|}{$\mathrm{T}(\mathrm{Rg})$} & \multicolumn{2}{|c|}{$\mathrm{T}(\mathrm{V})$} \\
\hline & Skin & Pulp & Skin & Pulp & Skin & Pulp & Skin & Pulp & Skin & Pulp \\
\hline \multicolumn{11}{|l|}{ C6 compounds } \\
\hline Hexanol & 287.6 & 172.9 & 67.7 & 72.5 & 94.9 & 99.9 & 105.2 & 76.9 & 277.3 & 395.8 \\
\hline trans-3-Hexenol & 22.2 & 14.2 & 1.9 & 5.1 & 4.6 & 8.8 & 3.9 & 4.6 & 5.3 & 11.3 \\
\hline cis-3-Hexenol & 73.7 & 52.3 & 23.2 & 19.6 & 15.8 & 10.8 & 22.4 & 11.7 & 27.9 & 50.1 \\
\hline trans-2-Hexenol & 62.0 & 24.0 & 22.6 & 14.4 & 30.2 & 9.7 & 27.5 & 11.7 & 21.1 & 32.8 \\
\hline Total & 445.5 & 263.4 & 115.4 & 111.6 & 145.5 & 129.2 & 159.0 & 104.9 & 331.6 & 490.0 \\
\hline \multicolumn{11}{|l|}{ Terpenes } \\
\hline trans-Furan linalool oxide & 24.9 & 25.5 & 9.7 & 5.1 & 7.0 & 6.2 & 14.1 & 8.8 & 4.9 & 15.5 \\
\hline cis-Furan linalool oxide & 19.9 & 17.2 & 5.2 & 4.0 & 4.9 & 4.1 & 11.4 & 7.3 & 6.8 & 13.1 \\
\hline trans-Pyran linalool oxide & 24.9 & 15.1 & 5.2 & 4.5 & 7.0 & 6.4 & 16.5 & 8.6 & 6.4 & 11.2 \\
\hline cis-Pyran linalool oxide & 16.4 & 20.9 & 4.2 & 4.6 & 4.9 & 6.6 & 15.7 & 11.4 & 9.8 & 25.1 \\
\hline Nerol & nd & 4.3 & nd & 1.5 & nd & 1.6 & nd & 1.1 & nd & 4.5 \\
\hline Geraniol & 39.8 & 20.0 & 19.3 & 8.5 & 7.0 & 8.3 & 9.4 & 7.7 & 7.5 & 30.1 \\
\hline Z-2,6-Dimethyl-2,7-octadiene-1,6-diol & 42.1 & 9.2 & 25.5 & 9.0 & 11.5 & 6.8 & 12.3 & 6.7 & 21.0 & 21.6 \\
\hline E-2,6-Dimethyl-2,7-octadiene-1,6-diol & 70.2 & 13.9 & 50.6 & 17.7 & 14.5 & 9.2 & 13.1 & 7.6 & 37.2 & 31.7 \\
\hline E-3,7-Dimethyl-2-en-1,7-octandiol & 16.4 & 13.9 & 18.5 & 10.9 & 6.4 & 8.8 & 8.5 & 9.1 & 24.6 & 30.7 \\
\hline Geranic acid & 70.2 & 5.5 & 20.7 & 3.9 & 6.4 & 4.0 & 7.7 & 4.6 & 17.5 & 10.1 \\
\hline p-Menthene-7-8-diol & 39.8 & nd & 8.8 & nd & 10.7 & nd & 12.3 & nd & 12.6 & nd \\
\hline Total & 364.6 & 145.5 & 167.7 & 69.7 & 80.3 & 62.0 & 121.0 & 72.9 & 148.3 & 193.6 \\
\hline \multicolumn{11}{|l|}{ Benzenoids } \\
\hline Benzaldehyde & 18.7 & 6.8 & 5.5 & 4.2 & 12.7 & 6.0 & 7.1 & 4.0 & 9.8 & 8.5 \\
\hline Methyl salicylate & 39.8 & 54.2 & 6.5 & 16.5 & 61.2 & 98.3 & 11.0 & 16.0 & 15.1 & 109.6 \\
\hline$\alpha$-Methyl-benzenemethanol & nd & 9.9 & nd & 0.9 & nd & 1.3 & nd & 1.6 & nd & 5.6 \\
\hline Eugenol & 12.9 & 16.6 & 3.1 & 5.4 & 4.3 & 8.3 & 2.7 & 4.8 & 5.6 & 18.4 \\
\hline Benzyl alcohol & 877.4 & 366.2 & 214.1 & 215.7 & 309.0 & 398.1 & 264.3 & 215.1 & 231.5 & 622.9 \\
\hline 2-phenylethanol & 596.7 & 230.8 & 134.8 & 104.5 & 158.2 & 153.0 & 213.0 & 118.0 & 164.0 & 330.9 \\
\hline Omovanillic alcohol & 238.7 & 80.0 & 35.2 & 25.3 & 51.2 & 28.8 & 54.7 & 54.2 & 56.5 & 165.6 \\
\hline Dihydroconiferyl alcohol & 28.1 & 29.2 & 7.0 & 12.2 & 12.8 & 12.2 & 23.1 & 18.2 & 14.0 & 48.8 \\
\hline Total & 1812.3 & 793.7 & 406.2 & 384.7 & 609.4 & 706.0 & 575.9 & 431.9 & 496.5 & 1310.3 \\
\hline \multicolumn{11}{|l|}{ Phenols } \\
\hline 4-Vinylphenol & 233.9 & 172.3 & 82.6 & 19.2 & 43.9 & 28.1 & 310.1 & 37.6 & 71.1 & 77.9 \\
\hline 4-Vinylguaicol & 11.7 & 97.9 & 2.2 & 20.8 & 4.3 & 27.3 & 6.9 & 24.2 & 6.3 & 177.6 \\
\hline Total & 245.6 & 270.2 & 84.4 & 40.0 & 48.2 & 55.4 & 317.0 & 61.8 & 77.4 & 255.5 \\
\hline \multicolumn{11}{|l|}{ Norisoprenoids } \\
\hline 3-Hydroxy- $\beta$-damascone ${ }^{a}$ & 49.1 & 34.8 & 14.1 & 17.9 & 17.9 & 13.2 & 21.6 & 13.8 & 9.8 & 65.9 \\
\hline $3-O \times 0-\alpha$-ionol ${ }^{\mathrm{b}}$ & 107.6 & 53.2 & 31.2 & 24.7 & 23.5 & 16.8 & 25.4 & 17.3 & 38.6 & 42.9 \\
\hline 3,9-Dihydroxy-mega-5-ene & 49.1 & 39.1 & 11.4 & 13.0 & 21.4 & 24.3 & 13.1 & 17.4 & 36.5 & 92.5 \\
\hline Vomifoliol $^{\mathrm{C}}$ & 400.1 & 131.7 & 151.7 & 72.8 & 129.0 & 71.8 & 132.1 & 99.6 & 164.0 & 292.0 \\
\hline Total & 605.9 & 258.8 & 208.4 & 128.4 & 191.8 & 126.1 & 192.2 & 148.1 & 248.9 & 493.3 \\
\hline
\end{tabular}

T, Trincadeira; (P), Portalegre; (B), Borba; (Rd), Redondo; (Rg), Reguengos; (V), Vidigueira.

a 3-Hydroxymegastigma-5,8-dien-7-one.

b 9-Hydroxymegastigma-4,7-dien-3-one.

c 6,9-Hihydroxymegastigma-4,7-dien-3-one.

C18 cartridges, was hydrolysed with $200 \mu \mathrm{L}$ of a commercial glycosidase rich enzyme (pectinol, Genencor International, Roncq, France) at $40^{\circ} \mathrm{C}$ for $24 \mathrm{~h}$; 1 heptanol was added as internal standard $\left(40 \mathrm{mg} \mathrm{L}^{-1}\right)$. This mixture, containing the aglycons released by enzymatic hydrolysis, was eluted through a Sep Pack ${ }^{\circledR}$ Cartridge C18 $1 \mathrm{~g}$ previously activated with $5 \mathrm{~mL}$ of methanol and $10 \mathrm{~mL}$ of water, and the free compounds recovered with $6 \mathrm{~mL}$ of dichloromethane. The dichloromethane extract was split in two. One volume, after drying under anhydrous sodium sulfate, was evaporated at ambient temperature and pressure. After concentration the sample was ready for GC/GC-MS analysis.
To the second volume of the dichloromethane extract $10 \mathrm{~mL}$ of tartaric buffer at $\mathrm{pH} 3.0$ and $1 \mathrm{~g}$ of sodium chloride were added. The mixture was kept in a water bath at $100^{\circ} \mathrm{C}$ for $1 \mathrm{~h}$. After cooling the sample (containing the product of chemical hydrolysis of varietal aromatic aglycones) was eluted through a Sep Pack ${ }^{\circledR}$ Cartridge C18 (300 mg), previously activated with $2 \mathrm{~mL}$ of methanol and $3 \mathrm{~mL}$ of water. After washing with $5 \mathrm{~mL}$ of water, the volatile compounds were eluted with $5 \mathrm{~mL}$ of dichloromethane, dried under anhydrous sodium sulfate and concentrated at ambient temperature and pressure prior to injection on $\mathrm{GC} / \mathrm{GC}-\mathrm{MS}$ system. 


\section{Standards chemicals and solvents}

All solvents/chemicals and standards were GC or pa grade from Sigma-Aldrich Co. (St Louis, MO, USA).

\section{Chromatographic conditions}

The capillary column was an Innovax (J\&W Scientific, Palo Alto, CA, USA) $30 \mathrm{~m} \times 0.25 \mathrm{~mm}$ d.i. and $0.25 \mu \mathrm{m}$ d.f. The gas chromatograph was a Hewlett Packard 5890 series II linked online with a selective detector MSD 5970 (Hewlett Packard, Wilmington, DE, USA); injector: $250^{\circ} \mathrm{C}$; interface $230^{\circ} \mathrm{C}$. The oven was programmed from $30^{\circ} \mathrm{C}$ for $2 \mathrm{~min}$, than linearly increased at a rate of $30^{\circ} \mathrm{C} \mathrm{min}^{-1}$ to $60^{\circ} \mathrm{C}$ and than at $2^{\circ} \mathrm{C} \mathrm{min}{ }^{-1}$ to $160^{\circ} \mathrm{C}$ and $3^{\circ} \mathrm{C} \mathrm{min}{ }^{-1}$ to $230^{\circ} \mathrm{C}$, and held for $13 \mathrm{~min}$. Carrier gas was helium: $70 \mathrm{kPa}$. The injection mode was splitless for $2 \mathrm{~min}$. Acquisition mass range was from 28 to 300 u.m.a.; ionization energy $70 \mathrm{eV}$. Compounds were identified by comparing retention times and mass spectra with standards, when available, or comparing the retention time and mass spectra with those reported in the literature. ${ }^{21-23}$ Semi-quantitative data were obtained by the ratio of peak height of individual compounds vs. internal standard peak height.

\section{RESULTS}

\section{Red varieties}

The volatile compounds released by enzymatic hydrolysis from the glycoside precursors in the Portuguese red grape varieties are shown in Table 1. Data show that, for all varieties, skins are richer than pulps.

Geraniol is the most abundant monooxygenated terpenic alcohol. Linalool and $\alpha$-terpineol were found in amounts lower then $1 \mu \mathrm{g} \mathrm{L}^{-1}$. Nerol was only quantified in pulps. Di-oxygenated compounds derived from linalool and geraniol are well represented, the most abundant being the furanic and pyranic oxides of linalool and the two isomers of 8hydroxylinalool. The $p$-ment-1-ene-7,8-diol, a very important aroma compound that derives from $\alpha$ terpineol, was found in important amounts in pulps from Tinta Caiada. Moreto variety seems to be the richer in terpenic compounds, followed by Castelão and Trincadeira. In Tinta Caiada the pyranic oxides of linalool were not found in detectable amounts.

The content of norisoprenoid compounds is higher in the Tinta Caiada variety, but all other varieties, except Trincadeira, have amounts greater than $400 \mu \mathrm{g} \mathrm{kg}^{-1}$.

The compounds produced by chemical hydrolysis of the aglycons obtained by enzymatic hydrolysis of the glycosylated precursors are presented in Table 2. The compounds obtained were easily identified by GC-MS. When these compounds are considered Moreto and Aragonez are the only varieties where pulps are richer than skins. Vitispirane isomers are the most representative compounds in Aragonez, and actinidol isomers in Moreto and Tinta Caiada. Moreto and Aragonez are also the richest varieties in Riesling acetal and TDN. Moreto is the richest variety in $\beta$-damascenone.

As far as soil is concerned we studied the same variety (Trincadeira) in eight different sub-regions. These results are presented in Table 1A for enzymatic hydrolysis from the glycosylated precursors from red grapes and in Table 2 for the compounds released by chemical hydrolysis.

For all samples skins are richer than pulps in terpenic and norisoprenoid compounds, once again with the exception of Vidigueira (Table 1A). Although there are some important differences in the amounts of compounds found in these grapes, the aromatic profile is very similar. As for the compounds produced by chemical hydrolysis of the aglycons generated by

Table 2. Compounds obtained by chemical hydrolysis from glycosylated precursors extracted from red grapes

\begin{tabular}{|c|c|c|c|c|c|c|c|c|c|c|}
\hline \multirow[b]{2}{*}{ Compounds $\left(\mu \mathrm{gg}^{-1}\right)$} & \multicolumn{2}{|c|}{$\mathrm{T}(\mathrm{E})$} & \multicolumn{2}{|c|}{$M(E)$} & \multicolumn{2}{|c|}{$\mathrm{TC}(\mathrm{E})$} & \multicolumn{2}{|c|}{$\mathrm{Az}(\mathrm{E})$} & \multicolumn{2}{|c|}{$C(E)$} \\
\hline & Pulp & Skin & Pulp & Skin & Pulp & Skin & Pulp & Skin & Pulp & Skin \\
\hline Vitispiranes & nd & 8.7 & 43.0 & 51.2 & 9.6 & 9.1 & 59.9 & 58.8 & 17.2 & 25.0 \\
\hline Riesling acetal & 0.9 & 13.5 & 87.9 & 20.2 & 1.5 & 6.5 & 28.9 & 21.7 & 10.6 & 15.1 \\
\hline TDN & 0.9 & 23.2 & 24.7 & 34.8 & 4.9 & 4.8 & 26.4 & 7.7 & 8.1 & 5.3 \\
\hline$\beta$-Damascenone & 4.3 & 6.3 & 16.5 & 27.5 & 9.3 & 4.3 & 11.5 & 3.6 & 8.6 & 4.5 \\
\hline \multirow[t]{2}{*}{ Actinidols } & 9.6 & 15.9 & 93.4 & 82.4 & 45.4 & 25.8 & 19.0 & 10.9 & 12.0 & 7.11 \\
\hline & \multicolumn{2}{|c|}{$\mathrm{T}(\mathrm{P})$} & \multicolumn{2}{|c|}{$\mathrm{T}(\mathrm{B})$} & \multicolumn{2}{|c|}{$\mathrm{T}(\mathrm{Rd})$} & \multicolumn{2}{|c|}{$\mathrm{T}(\mathrm{Rg})$} & \multicolumn{2}{|c|}{$\mathrm{T}(\mathrm{V})$} \\
\hline Compounds $\left(\mu \mathrm{kg}^{-1}\right)$ & Pulp & Skin & Pulp & Skin & Pulp & Skin & Pulp & Skin & Pulp & Skin \\
\hline Vitispiranes & 10.9 & 27.1 & 6.9 & 6.6 & 8.7 & nd & 8.9 & nd & 8.4 & 9.9 \\
\hline Riesling acetal & 27.4 & 10.2 & 7.1 & 7.2 & 14.9 & 2.3 & 8.9 & 7.1 & 6.9 & 5.3 \\
\hline TDN & 5.5 & 25.9 & 7.7 & 4.4 & 7.5 & nd & 1.6 & 2.9 & 6.1 & 8.0 \\
\hline$\beta$-Damascenone & 18.3 & 16.6 & 5.3 & 3.7 & 4.2 & 4.1 & 4.1 & 3.3 & 7.6 & 15.2 \\
\hline Actinidols & 21.9 & 21.5 & 12.4 & 11.1 & 15.4 & 9.9 & 6.8 & 12.3 & 4.6 & 22.4 \\
\hline
\end{tabular}

T, Trincadeira; M, Moreto; TC, Tinta Caiada; Az, Aragonez; C, Castelão; E, Évora; P, Portalegre; B, Borba: Rd, Redondo; Rg, Reguengos; V, Vidigueira. 
enzymatic hydrolysis (Table 2), Trincadeira seems to be poor in precursors of vitispirane isomers, Riesling acetal, TDN, damascenone and actinidol isomers, but the ratios between these compounds, however, remain the same in different environments.

\section{White varieties}

As can be observed in Table 3, skins are richer than pulps, as expected. Arinto has highest values of terpenes. In all these varieties we found more compounds from the linalool class, with cis-8-hydroxy-linalool being the most important. Arinto variety, however, shows a high level of compounds from the geraniol class. Nevertheless all grape varieties have an important terpenic component that can be expressed in aromatic notes depending on the ratio linalool:geraniol.

As far as content of norisoprenoids is concerned, they are poorly represented, with vomifoliol being the most important. Arinto is richer in norisoprenoid compounds and Roupeiro is poorest. From the results in Table 4 we see that several differences

Table 3. Compounds released by enzymatic hydrolysis from the glycosylated precursors extracted from white grapes

\begin{tabular}{|c|c|c|c|c|c|c|c|c|c|c|}
\hline \multirow[b]{2}{*}{ Compounds $\left(\mu \mathrm{g} \mathrm{kg}^{-1}\right)$} & \multicolumn{2}{|c|}{$\mathrm{R}$} & \multicolumn{2}{|c|}{$A v$} & \multicolumn{2}{|c|}{$\mathrm{RO}$} & \multicolumn{2}{|c|}{$\mathrm{Pr}$} & \multicolumn{2}{|c|}{ A } \\
\hline & Skin & Pulp & Skin & Pulp & Skin & Pulp & Skin & Pulp & Skin & Pulp \\
\hline \multicolumn{11}{|l|}{ C6 compounds } \\
\hline Hexanol & 57.4 & 49.2 & 43.2 & 31.9 & 29.2 & 38.1 & 15.7 & 12.2 & 79.1 & 19.0 \\
\hline cis-3-Hexenol & 9.8 & 7.9 & 17.2 & 9.2 & 9.1 & 13.6 & 9.6 & 5.4 & 6.4 & 1.8 \\
\hline Trans-2-Hexenol & 5.9 & 6.7 & 11.6 & 6.6 & 3.2 & 4.2 & 5.1 & 1.6 & 27.5 & 1.5 \\
\hline Total & 73.1 & 63.8 & 72 & 47.7 & 41.5 & 55.9 & 30.4 & 19.2 & 113 & 22.3 \\
\hline \multicolumn{11}{|l|}{ Terpenes } \\
\hline trans-Furan linalool oxide & 11.9 & 10.5 & 9.4 & 7.2 & 12.3 & 9.7 & 11.2 & 7.3 & 19.4 & 3.7 \\
\hline cis-Furan linalool oxide & 9.5 & 11.1 & 8.9 & 5.8 & 7.8 & 8.1 & 9.3 & 10.0 & 6.4 & 2.3 \\
\hline Linalool & 58.2 & 26.8 & 13.9 & 4.1 & 70.7 & 31.4 & 6.1 & 1.4 & 75.1 & 4.9 \\
\hline$\alpha$-Terpineol & 11.9 & 3.8 & nd & nd & 4.5 & 1.9 & nd & nd & 4.4 & 2.1 \\
\hline trans-Pyran linalool oxide & 8.9 & 7.5 & 8.3 & 5.9 & 10.4 & 9.7 & 1.9 & 1.9 & 6.4 & 1.6 \\
\hline cis-Pyran linalool oxide & 10.7 & 9.8 & 10.5 & 6.9 & 13.6 & 13.6 & 16.6 & 12.0 & 12.1 & 2.3 \\
\hline Nerol & 1.8 & 2.7 & 26.7 & 6.3 & 8.4 & 5.3 & 4.5 & 3.1 & 9.7 & 1.1 \\
\hline Geraniol & 14.9 & 10.1 & 49.9 & 17.3 & 34.4 & 40.4 & 22.4 & 23.9 & 64.6 & 5.0 \\
\hline E-3,7-Dimethyl-3,7-dihydroxi-1,5-octadiene & 10.1 & 25.9 & 10.5 & 15.1 & 13.7 & 25.2 & 8.6 & 17.6 & 25.0 & 11.6 \\
\hline 3,7-Dimethyl-3,7-dihydroxi-1-octene & 4.2 & 7.0 & 4.4 & 4.3 & 6.9 & 3.4 & 3.2 & 6.0 & 14.5 & 7.0 \\
\hline 3,7-Dimethyl-1,7-octadiol & 9.9 & 0.9 & 9.3 & 1.9 & 22.7 & 7.5 & 9.0 & 1.3 & 158.4 & 9.3 \\
\hline 2,6-Dimethyl-7-octane-1,6-diol & 7.3 & 6.2 & 10.2 & 8.4 & 21.3 & 20.5 & 7.6 & 2.1 & 47.5 & 8.6 \\
\hline Z-2,6-Dimethyl-2,7-octadiene-1,6-diol & 36.0 & 21.6 & 63.0 & 16.9 & 59.7 & 53.9 & 22.2 & 8.1 & 82.4 & 12.1 \\
\hline E-2,6-Dimethyl-2,7-octadiene-1,6-diol & 64.8 & 81.1 & 143.4 & 47.6 & 259.9 & 81.2 & 142.0 & 37.6 & 285.0 & 60.2 \\
\hline E-3,7-Dimethyl-2-en-1,7-octandiol & 23.9 & 14.2 & nd & 9.1 & 47.7 & 13.0 & nd & 12.4 & 161.1 & 19.6 \\
\hline Geranic acid & 59.3 & 6.3 & 107.5 & 2.8 & 37.8 & 11.6 & 37.4 & 5.9 & 158.4 & 3.9 \\
\hline p-Menthene-7,8-diol & 26.4 & 9.0 & 16.1 & 3.9 & 9.6 & nd & 25.6 & 7.3 & 53.7 & 9.3 \\
\hline Total & 369.7 & 254.5 & 492 & 163.5 & 641.4 & 336.4 & 327.6 & 157.9 & 1184 & 164.6 \\
\hline \multicolumn{11}{|l|}{ Benzenoids } \\
\hline Benzaldehyde & 3.3 & 2.8 & 8.3 & 2.0 & 4.5 & 13.1 & 4.5 & 1.4 & 7.3 & 1.5 \\
\hline Methyl salicylate & 27.9 & 81.1 & 11.1 & 11.3 & 3.2 & 15.3 & 2.2 & 1.4 & 8.1 & 3.0 \\
\hline$\alpha$-Methyl-benzenemethanol & 1.5 & 2.4 & 3.3 & 2.1 & 1.9 & 2.5 & 1.6 & 1.5 & 4.0 & 1.2 \\
\hline Benzyl alcohol & 200.7 & 209.4 & 143.4 & 122.5 & 294.4 & 262.5 & 164.0 & 103.7 & 279.9 & 92.5 \\
\hline 2-Phenylethanol & 191.2 & 152.7 & 128.5 & 86.2 & 175.0 & 172.8 & 143.3 & 91.7 & 364.3 & 88.2 \\
\hline Omovanillic alcohol & 31.6 & 45.9 & 70.4 & 60.1 & 98.1 & nd & 118.8 & 101.0 & 31.7 & 18.2 \\
\hline Dihydroconiferyl alcohol & 6.6 & 14.8 & 24.7 & 7.8 & nd & 121.2 & 9.7 & 16.6 & 9.6 & 8.7 \\
\hline Total & 462.8 & 509.1 & 389.7 & 292 & 577.1 & 587.4 & 444.1 & 317.3 & 704.9 & 213.3 \\
\hline \multicolumn{11}{|l|}{ Phenols } \\
\hline 4-vinylguaicol & 2.2 & 43.5 & 6.7 & 39.3 & 13.7 & 69.6 & 10.4 & 103.8 & 14.3 & 32.2 \\
\hline 4-vinylphenol & 12.8 & 16.4 & 11.7 & 8.1 & 9.6 & 9.5 & 5.5 & 7.1 & 23.8 & 36.2 \\
\hline Total & 15 & 59.9 & 18.4 & 47.4 & 23.3 & 79.1 & 15.9 & 110.9 & 38.1 & 68.4 \\
\hline \multicolumn{11}{|l|}{ Norisoprenoids } \\
\hline 3-Hydroxy- $\beta$-damascone ${ }^{a}$ & 18.5 & 15.8 & 43.3 & 19.9 & 6.9 & nd & 31.2 & 17.4 & 82.5 & 21.2 \\
\hline $3-O \times 0-\alpha-$ ionol $^{\mathrm{b}}$ & 16.5 & 10.1 & 95.0 & 26.2 & 58.3 & 31.4 & 27.0 & 15.7 & 31.7 & 12.0 \\
\hline 3,9-Dihydroxy-mega-5-ene & 20.2 & 12.9 & 30.5 & 41.3 & 3.4 & nd & 46.4 & 29.9 & 88.7 & 17.4 \\
\hline Vomifoliol $^{\mathrm{C}}$ & 101.1 & 52.6 & 128.5 & 76.5 & 169.7 & 85.3 & 93.0 & 66.6 & 322.1 & 62.9 \\
\hline Total & 156.3 & 91.4 & 297.3 & 163.9 & 238.3 & 116.7 & 197.6 & 129.6 & 525 & 113.5 \\
\hline
\end{tabular}

R, Roupeiro; AV, Antão Vaz; RO, Rabo de Ovelha; Pr, Perrum; A, Arinto; (E), Évora.

a 3-Hydroxymegastigma-5,8-dien-7-one.

b 9-Hydroxymegastigma-4,7-dien-3-one.

c 6,9-Dihydroxymegastigma-4,7-dien-3-one. 
Table 3A. Compounds released by enzymatic hydrolysis from the glycosylated precursors extracted from white grapes

\begin{tabular}{|c|c|c|c|c|c|c|c|c|c|c|}
\hline \multirow[b]{2}{*}{ Compounds ( $\left.\mu \mathrm{g} \mathrm{kg}^{-1}\right)$} & \multicolumn{2}{|c|}{$R(P)$} & \multicolumn{2}{|c|}{$\mathrm{R}(\mathrm{B})$} & \multicolumn{2}{|c|}{$\mathrm{R}(\mathrm{Rd})$} & \multicolumn{2}{|c|}{$R(R g)$} & \multicolumn{2}{|c|}{$R(V)$} \\
\hline & Skin & Pulp & Skin & Pulp & Skin & Pulp & Skin & Pulp & Skin & Pulp \\
\hline \multicolumn{11}{|l|}{ C6 compounds } \\
\hline Hexanol & 55.0 & 49.7 & 36.2 & 23.8 & 56.3 & 49.4 & 67.2 & 38.0 & 97.6 & 61.6 \\
\hline cis-3-Hexenol & 15.4 & 12.0 & 7.3 & 3.6 & 10.5 & 9.6 & 17.7 & 7.1 & 19.2 & 11.5 \\
\hline Trans-2-Hexenol & 9.5 & 8.6 & 12.3 & 11.7 & 4.3 & 5.5 & 7.2 & 5.0 & 9.2 & 4.4 \\
\hline Total & 79.9 & 70.3 & 55.8 & 39.1 & 71.1 & 64.5 & 92.1 & 50.1 & 126.0 & 77.5 \\
\hline \multicolumn{11}{|l|}{ Terpenes } \\
\hline trans-Furan linalool oxide & 12.8 & 14.3 & 7.8 & 4.2 & 12.1 & 11.8 & 15.2 & 5.9 & 17.0 & 8.4 \\
\hline cis-Furan linalool oxide & 12.5 & 14.9 & 5.3 & 2.9 & 11.8 & 12.8 & 15.9 & 6.4 & 16.3 & 10.0 \\
\hline Linalool & 86.6 & 42.0 & 13.9 & 3.2 & 57.3 & 26.3 & 27.4 & 8.4 & 43.3 & 14.0 \\
\hline$\alpha$-Terpineol & 5.9 & 4.1 & 1.7 & 1.3 & 16.6 & 3.8 & 7.5 & 2.2 & 11.4 & 3.3 \\
\hline trans-Pyran linalool oxide & 10.3 & 8.6 & 6.7 & 2.6 & 9.0 & 8.0 & 13.0 & 5.5 & 15.3 & 7.0 \\
\hline cis-Pyran linalool oxide & 13.2 & 13.4 & 7.3 & 2.9 & 12.5 & 9.9 & 18.1 & 6.9 & 23.1 & 14.8 \\
\hline Nerol & nd & 3.3 & nd & 0.3 & 2.0 & 5.2 & nd & 1.4 & 5.7 & 2.8 \\
\hline Geraniol & 30.8 & 9.2 & 9.5 & 2.9 & 30.2 & 9.6 & 14.4 & 5.5 & 19.2 & 8.4 \\
\hline E-3,7-Dimethyl-3,7-dihydroxi-1,5-octadiene & 13.9 & 25.8 & 5.0 & 3.6 & 10.9 & 23.4 & 4.3 & 5.5 & 9.9 & 12.2 \\
\hline 3,7-Dimethyl-3,7-dihydroxi-1-octene & 7.3 & 3.5 & 1.7 & 2.1 & 1.1 & 2.9 & 2.9 & 5.0 & 3.6 & 4.2 \\
\hline 3,7-Dimethyl-1,7-octadiol & 8.8 & 2.0 & 4.0 & 0.8 & 8.3 & 1.0 & 5.8 & 1.3 & 6.9 & 1.6 \\
\hline 2,6-Dimethyl-7-octane-1,6-diol & 10.4 & 6.8 & 8.1 & 1.8 & 8.0 & 7.2 & 8.7 & 4.6 & 13.0 & 7.2 \\
\hline Z-2,6-Dimethyl-2,7-octadiene-1,6-diol & 41.1 & 22.7 & 26.3 & 8.3 & 32.0 & 18.3 & 4.1 & 16.3 & 51.5 & 18.6 \\
\hline E-2,6-Dimethyl-2,7-octadiene-1,6-diol & 83.7 & 82.6 & 28.2 & 17.9 & 34.8 & 63.6 & 12.0 & 79.1 & 90.5 & 87.5 \\
\hline E-3,7-Dimethyl-2-en-1,7-octandiol & 71.1 & 11.1 & 14.1 & 6.6 & 24.2 & 13.0 & 24.8 & 18.2 & 31.1 & 17.9 \\
\hline Geranic acid & 85.3 & 1.6 & 36.3 & 3.7 & 47.3 & 2.7 & 62.3 & 5.3 & 81.6 & 5.3 \\
\hline p-Menthene-7,8-diol & 32.5 & 14.0 & 23.0 & 6.1 & 23.0 & 10.7 & 22.5 & 11.2 & 35.6 & 16.7 \\
\hline Total & 526.2 & 279.9 & 198.9 & 71.2 & 341.1 & 230.2 & 258.9 & 188.7 & 475.0 & 239.9 \\
\hline \multicolumn{11}{|l|}{ Benzenoids } \\
\hline Benzaldehyde & 1.5 & 3.7 & 1.4 & 2.2 & 4.9 & 4.8 & 2.2 & 3.2 & 2.1 & 3.9 \\
\hline Methyl salicylate & 6.6 & 22.3 & 15.6 & 30.5 & 32.7 & 59.6 & 9.4 & 20.1 & 7.1 & 14.8 \\
\hline$\alpha$-Methyl-benzenemethanol & 3.7 & 5.9 & 2.2 & 1.7 & 2.2 & 4.3 & 2.2 & 1.4 & 1.4 & 2.1 \\
\hline Benzyl alcohol & 202.1 & 243.7 & 230.6 & 205.0 & 200.3 & 209.5 & 204.5 & 178.0 & 227.1 & 237.9 \\
\hline 2-Phenylethanol & 176.9 & 134.8 & 163.4 & 95.4 & 147.3 & 141.4 & 164.6 & 111.1 & 211.2 & 158.6 \\
\hline Omovanillic alcohol & 66.3 & 33.3 & 45.4 & 17.8 & 30.8 & 40.7 & 55.9 & 53.4 & 67.4 & 70.8 \\
\hline Dihydroconiferyl alcohol & 33.2 & 10.4 & 14.1 & 9.5 & 5.0 & 4.6 & 9.1 & 9.6 & 10.8 & 12.4 \\
\hline Total & 490.3 & 454.1 & 472.7 & 362.1 & 423.2 & 464.9 & 447.9 & 376.8 & 527.1 & 500.5 \\
\hline \multicolumn{11}{|l|}{ Phenols } \\
\hline 4-Vinylguaicol & 4.0 & 44.9 & 3.6 & 24.9 & 1.9 & 31.8 & 1.7 & 53.5 & 4.6 & 53.2 \\
\hline 4-Vinylphenol & 17.5 & 12.0 & 14.5 & 14.3 & 9.4 & 6.8 & 19.0 & 11.5 & 16.6 & 18.1 \\
\hline Total & 21.5 & 56.9 & 18.1 & 39.2 & 11.3 & 38.6 & 20.7 & 65.0 & 21.2 & 71.3 \\
\hline \multicolumn{11}{|l|}{ Norisoprenoids } \\
\hline 3-Hydroxy- $\beta$-damascone ${ }^{a}$ & 14.4 & 28.7 & 13.7 & 10.9 & 17.7 & 16.6 & 14.5 & 22.7 & 18.6 & 26.8 \\
\hline $3-\mathrm{O} \times \mathrm{x}-\alpha-$-ionol $^{\mathrm{b}}$ & 30.0 & 17.8 & 45.6 & 10.1 & 25.3 & 10.8 & 20.7 & 12.8 & 24.0 & 11.4 \\
\hline 3,9-Dihydroxy-mega-5-ene & 8.0 & 12.4 & 8.1 & 6.7 & 19.3 & 14.6 & 15.3 & 12.0 & 24.8 & 14.7 \\
\hline Vomifoliol' $^{\mathrm{C}}$ & 140.5 & 61.2 & 143.5 & 46.1 & 97.3 & 33.9 & 135.8 & 47.8 & 142.0 & 57.8 \\
\hline Total & 129.9 & 120.1 & 210.9 & 73.8 & 159.6 & 75.9 & 186.3 & 95.3 & 209.4 & 110.7 \\
\hline
\end{tabular}

R, Roupeiro; (P), Portalegre; (B), Borba; (Rd), Redondo; (Rg), Reguengos; (V), Vidigueira.

a 3-Hydroxymegastigma-5,8-dien-7-one.

b 9-Hydroxymegastigma-4,7-dien-3-one.

c 6,9-Dihydroxymegastigma-4,7-dien-3-one.

can be observed among the varieties but, as noticed before, the greatest differences are between pulp and skin amounts rather then variety and/or region.

When soil is considered (Table $3 \mathrm{~A}$ ) the ratios found between the isomers remain the same in all sub-regions and as observed before skins are richer then pulps. Differences among the different sub-regions can be observed when the amounts of individuals is considered. The differences, however, lie in the intervals attributed before for the varieties.
The same is true when the norisoprenoid compounds are considered.

Similar results are obtained when the compounds generated by acid hydrolysis of the aglycons produced by enzymatic hydrolysis of the bound aromatic compounds (Table 4) are evaluated.

\section{DISCUSSION AND CONCLUSIONS}

Bound aroma compounds, namely terpenes and norisoprenoids, of ten Portuguese varieties of grapes 
Table 4. Compounds obtained by chemical hydrolysis from glycosylated precursors extracted from white grapes

\begin{tabular}{|c|c|c|c|c|c|c|c|c|c|c|}
\hline \multirow[b]{2}{*}{ Compounds $\left(\mu \mathrm{g} \mathrm{kg}^{-1}\right)$} & \multicolumn{2}{|c|}{$R(E)$} & \multicolumn{2}{|c|}{$\mathrm{AV}(\mathrm{E})$} & \multicolumn{2}{|c|}{$\mathrm{RO}(\mathrm{E})$} & \multicolumn{2}{|c|}{$\operatorname{Pr}(E)$} & \multicolumn{2}{|c|}{$A(E)$} \\
\hline & Pulp & Skin & Pulp & Skin & Pulp & Skin & Pulp & Skin & Pulp & Skin \\
\hline Vitispiranes & 31.8 & 29.5 & 40.9 & 16.0 & 10.0 & 11.1 & 41.3 & 56.3 & 26.2 & 67.4 \\
\hline Riesling acetal & 27.4 & 25.9 & 15.6 & 35.1 & 20.5 & 5.2 & 15.6 & 66.3 & 4.3 & 44.9 \\
\hline TDN & 21.9 & 11.8 & 15.3 & 8.2 & 4.1 & 4.7 & 15.4 & 11.9 & 3.1 & 15.0 \\
\hline$\beta$-Damascenone & 5.4 & 1.8 & 5.5 & 7.8 & nd & 7.0 & 7.5 & 10.4 & 9.0 & 13.5 \\
\hline \multirow[t]{2}{*}{ Actinidols } & 12.4 & 10.0 & 34.6 & 75.3 & 51.5 & 79.9 & 14.5 & 25.2 & 57.8 & 107.8 \\
\hline & \multicolumn{2}{|c|}{$R(P)$} & \multicolumn{2}{|c|}{$\mathrm{R}(\mathrm{B})$} & \multicolumn{2}{|c|}{$\mathrm{R}(\mathrm{Rd})$} & \multicolumn{2}{|c|}{$\mathrm{R}(\mathrm{Rg})$} & \multicolumn{2}{|c|}{$R(V)$} \\
\hline Compounds $\left(\mu \mathrm{g} \mathrm{kg}^{-1}\right)$ & Pulp & Skin & Pulp & Skin & Pulp & Skin & Pulp & Skin & Pulp & Skin \\
\hline Vitispiranes & 9.6 & 20.9 & 17.0 & 11.3 & 20.2 & 20.1 & 49.5 & 25.7 & 52.4 & 35.9 \\
\hline Riesling acetal & 10.3 & 6.8 & 9.5 & 13.7 & 28.3 & 18.0 & 30.4 & 18.2 & 31.5 & 22.2 \\
\hline TDN & 2.1 & 5.2 & 5.0 & 12.1 & 10.8 & 11.3 & 24.7 & 23.6 & 23.2 & 20.4 \\
\hline$\beta$-Damascenone & 2.7 & 8.5 & 1.9 & 6.0 & 3.2 & 6.4 & 3.2 & 6.4 & 3.0 & 7.2 \\
\hline Actinidols & 11.0 & 19.8 & 7.6 & 18.2 & 10.1 & 15.2 & 12.7 & 15.5 & 9.7 & 19.5 \\
\hline
\end{tabular}

R, Roupeiro; AV, Antão Vaz; RO, Rabo de Ovelha; Pr, Perrum; A, Arinto; E, Évora; P, Portalegre; B, Borba: Rd, Redondo; Rg, Reguengos; V, Vidigueira.

were studied. The same aroma compounds as in aromatic grapes were found, namely terpenes, benzenoids and norisoprenoids, but the amounts were rather small. Geraniol, linalool and $\alpha$-terpineol, the dominant terpenes in Muscat varieties (aromatic grapes),${ }^{9}$ did not show relevant amounts in all cases. In fact they were all present in the white varieties but not in the red ones. Chemical hydrolysis of norisoprenoid aglycons released by enzymatic hydrolyses produced vitispirane isomers, actinidol isomers, Riesling acetal, TDN and $\beta$-damascenone. In some varieties skins are richer than pulps, which is not usually stated. ${ }^{7}$ In some cases amounts of $\mathrm{C} 13$ norisoprenoid aglycones are higher than the usual amounts, which rarely reach $100 \mu \mathrm{g} \mathrm{L}^{-1}$.

If the aroma impact of these results was to be estimated, however, we could more or less consider that the amounts released still remain far below the usually detected amounts in aromatic grapes, where monoterpene concentrations can reach $14 \mathrm{mg} \mathrm{L}^{-1}$.

Results obtained indicate some differences in syntheses of varietal glycosylated aroma compounds among the Portuguese red and white grape varieties studied. Differences seem to be higher in white varieties. The differences concern terpenic,

Table 5. Ratios between aroma compounds of white grapes pulps and skins

\begin{tabular}{|c|c|c|c|c|c|c|c|c|c|c|}
\hline & RE & $\mathrm{RRd}$ & $\mathrm{RP}$ & $\mathrm{RB}$ & RV & $\mathrm{RRg}$ & AV & $A$ & $\mathrm{Pr}$ & $\mathrm{RO}$ \\
\hline \multicolumn{11}{|l|}{ Pulps } \\
\hline OxA/oxB & $<1$ & $<1$ & $<1$ & $<1$ & $<1$ & $<1$ & & & $<1$ & \\
\hline OxC/oxD & $<1$ & $<1$ & $<1$ & $<1$ & $<1$ & $<1$ & $<1$ & $<1$ & $<1$ & $<1$ \\
\hline T8OHlin/C8Ohlin & $<1$ & $<1$ & $<1$ & $<1$ & $<1$ & $<1$ & $<1$ & $<1$ & $<1$ & $<1$ \\
\hline $\begin{array}{l}3 \mathrm{OH} \beta \text { dam/30xo } \alpha \text { ion } \\
\text { alc.Omo./diidrocon } \\
(\mathrm{T} 8 \mathrm{OH}+\mathrm{C} 8 \mathrm{OH}) / \text { pment } \\
\text { Lin/ } \alpha \text { terp }\end{array}$ & & & & & & & $<1$ & & & \\
\hline Lin/ger & & & & & & & $<1$ & $<1$ & $<1$ & $<1$ \\
\hline \multicolumn{11}{|l|}{ Skins } \\
\hline OxA/oxB & & & & & & $<1$ & & & & \\
\hline OxC/OxD & $<1$ & $<1$ & $<1$ & $<1$ & $<1$ & $<1$ & $<1$ & $<1$ & & $<1$ \\
\hline T8OHlin/C8Ohlin & $<1$ & $<1$ & $<1$ & $<1$ & $<1$ & $<1$ & $<1$ & $<1$ & $<1$ & $<1$ \\
\hline $\begin{array}{l}3 \mathrm{OH} \beta \text { dam/3oxo } \alpha \text { ion } \\
\text { alc.Omo./diidrocon }\end{array}$ & & $<1$ & $<1$ & $<1$ & $<1$ & $<1$ & $<1$ & & $<1$ & $<1$ \\
\hline $\begin{array}{l}(\mathrm{T} 8 \mathrm{OH}+\mathrm{C} 8 \mathrm{OH}) / \text { pment } \\
\mathrm{Lin} / \alpha \text { terp }\end{array}$ & & & & & & $<1$ & & & & \\
\hline Lin/ger & & & & & & & $<1$ & & $<1$ & \\
\hline
\end{tabular}

RV, Roupeiro (Vidigueira); RRd, Roupeiro (Redondo); RRg, Roupeiro (Reguengos); RB, Roupeiro (Borba); RP, Roupeiro (Portalegre); RE, Roupeiro (Évora); AV, Antão Vaz; RO, Rabo de Ovelha; Pr, Perrum; A, Arinto.

OxA, trans isomer of the furanic oxide of linalool; OxB, cis isomer of the furanic oxide of linalool; lin, linalol; $\alpha$ terp, $\alpha$-terpineol OxC, trans isomer of the pyranic oxide of linalool; OxD, cis isomer of the pyranic oxide of linalool; ger, geraniol; T8OHlin, $Z$ isomer of 2,6-dimethyl-2,7-octadiene-1,6-diol; $\mathrm{C} 8 \mathrm{OH}$ lin, $E$ isomer of 2,6-dimethyl-2,7-octadiene-1,6-diol; pment, $p$-menthene-7,8-diol; alc.Omo., 4-hydroxy-3-methoxyphenethanol; diidrocon, dihydroconipherilic alcohol; $3 \mathrm{OH} \beta$ dam, 3-hydroxy- $\beta$-damascone; 3oxo $\alpha$ ion, 3-oxo- $\alpha$-ionol (one of the compounds was not detected). 
Table 6. Ratios between aroma compounds of red grapes pulps and skins

\begin{tabular}{|c|c|c|c|c|c|c|c|c|c|c|}
\hline & $\mathrm{TV}$ & TRd & $\mathrm{TRg}$ & $\mathrm{TB}$ & $\mathrm{TP}$ & TE & $\mathrm{TC}$ & $\mathrm{Az}$ & $\mathrm{C}$ & $\mathrm{M}$ \\
\hline \multicolumn{11}{|l|}{ Pulps } \\
\hline OxA/oxB & & & & & & & & $<1$ & & $<1$ \\
\hline $\mathrm{OxC} / \mathrm{oxD}$ & $<1$ & $<1$ & $<1$ & $<1$ & $<1$ & & & $<1$ & $<1$ & $<1$ \\
\hline T8OHlin/C8OHlin & $<1$ & $<1$ & $<1$ & $<1$ & $<1$ & $<1$ & & $<1$ & $<1$ & $<1$ \\
\hline $\begin{array}{l}\text { 3ОH } \beta \text { dam/3oxo } \alpha \text { ion } \\
\text { alc.Omo./diidrocon } \\
(\mathrm{T} 8 \mathrm{OH}+\mathrm{C} 8 \mathrm{OH}) / \text { pment }\end{array}$ & & $<1$ & $<1$ & $<1$ & $<1$ & $<1$ & & $<1$ & & $\begin{array}{l}<1 \\
<1\end{array}$ \\
\hline \multicolumn{11}{|l|}{ Skins } \\
\hline OxA/oxB & $<1$ & & & & & & & $<1$ & & $<1$ \\
\hline $\mathrm{OxC} / \mathrm{oxD}$ & $<1$ & & & & & & & $<1$ & & $<1$ \\
\hline T8OHlin/C8OHlin & $<1$ & $<1$ & $<1$ & $<1$ & $<1$ & $<1$ & $<1$ & $<1$ & & $<1$ \\
\hline $\begin{array}{l}\text { 30H } \beta \text { dam/3oxo } \alpha \text { ion } \\
\text { alc.Omo./diidrocon }\end{array}$ & $<1$ & $<1$ & $<1$ & $<1$ & $<1$ & $<1$ & & $<1$ & $<1$ & $<1$ \\
\hline$(\mathrm{T} 8 \mathrm{OH}+\mathrm{C} 8 \mathrm{OH}) / \mathrm{pment}$ & & & & & & & $<1$ & & & \\
\hline
\end{tabular}

TV, Trincadeira (Vidigueira); TRd, Trincadeira (Redondo); TRg, Trincadeira (Reguengos); TB, Trincadeira (Borba); TP, Trincadeira (Portalegre); TE, Trincadeira (Évora); M, Moreto; TC, Tinta Caiada; Az, Aragonez; C, Castelão.

OxA, trans isomer of the furanic oxide of linalool; OxB, cis isomer of the furanic oxide of linalool; OxC, trans isomer of the pyranic oxide of linalool; OxD, cis isomer of the pyranic oxide of linalool; T8OHlin, $Z$ isomer of 2,6-dimethyl-2,7-octadiene-1,6-diol; C8OHlin, $E$ isomer of 2,6-dimethyl-2,7octadiene-1,6-diol; pment, $p$-menthene-7,8-diol; alc.Omo., homovanilic alcohol; diidrocon, dihydroconipherilic alcohol; one of the compounds was not detected.

norisoprenoid and benzenoid compound amounts as well as ratios between isomeric compounds. The ratios studied are those usually used to classify the varieties according to their potential flavour. ${ }^{1}$ When these specific ratios are evaluated (Tables 5 and 6) it can be concluded that all the grapes studied can be considered neutral.

Another volatile compound also found in all varieties was $p$-menthene-7,8-diol. This result is in agreement with similar data that consider this compound the one that usually prevails in grapes. $^{24}$

An influence of different environments in the two varieties studied can probably be outlined, although study has to be extended over several years to be conclusive. Qualitative profile and ratios between isomeric compounds remain more or less the same regardless of the sub-region (Tables 5 and 6). This fact should be explored to search for a possible varietal discrimination. When quantitative data are considered differences are noticed (Tables 1A, 2, $3 \mathrm{~B}$, and 4 ), which is in agreement with previous results. $^{5,9,11}$

The technological impact of this study is yet far from being concluded; as a first approach we can consider that the enzymatic and acidic hydrolysis followed by a higher skin contact (especially for the white varieties) can improve/enhance wine aroma. By increasing the concentrations of free monoterpenes in wine as well as other potent odorants such as norisoprenoids, the varietal aroma and hence perceived quality can also be increased. The impact of the early release of these compounds on wine ageing has to be evaluated as well as contact time to avoid deleterious effect of phenols and/or rearrangement of the monoterpenes liberated under the mild acidic conditions of wine ${ }^{13}$.

\section{ACKNOWLEDGEMENTS}

The authors wish to thank PAMAF 6007 for financial support; Maria João Cabrita wishes to thank the Istituto Sperimental per L'Enologia, Asti, Italy, for receiving her for a short stay.

\section{REFERENCES}

1 Di Stefano R, Metodi chimici nella caratterizzazione varietale: valutazioni attraverso lo studio dei composti volatili liberi e legati. In Annali dell'Istituto Sperimentale per l'Enologia Asti, Vol. XXVII, pp. 33-49 (1996).

2 Williams PJ, Sefton MA and Francis IL, Glycosidic precursors of varietal grape and wine flavour, in Flavor Precursors: Thermal and Enzymatic Conversions, ed. by Teranishi R, Takeoka GR and Guntert M. American Chemical Society, New York (1991).

3 Sefton MA, Francis IL and Williams PJ, Free and bound volatile secondary metabolities of Vitis vinifera grape cv. Sauvignon blanc. f Food Sci 59:142-147 (1994).

4 Sefton MA, Francis IL and Williams PJ, The volatile composition of Chardonnay juices: a study by flavor precursor analysis Am F Enol Vitic 44:359-370 (1993).

5 Rapp A, Volatile flavour of wine: correlation between instrumental analysis and sensory perception. Nahrung 42:351-363 (1998).

6 Vernin G, Les composants volatils des arômes de vin et de jus de raisin. Parfums, cosmétiques, arômes 68:83-93 (1986).

7 Baek $\mathrm{HH}$ and Cadwallader, KR Contribution of free and glycosidically bound volatile compounds to the aroma of Muscadine grape juice. F Food Sci 64:441-444 (1999).

8 Gunata Z, Bitteur S, Baumes R, Sapis JC and Bayonove $\mathrm{C}$, Activités glycosidases en vinification: perspectives d'exploitation des précurseurs d'arome du raisin, de nature glycosidique. Rev Fr Oenol. 122:37-41 (1990).

9 Mateo JJ and Jiménez M, Monoterpenes in grape juice and wine. f Chromatogr A 881:557-567 (2000).

10 Baumes R, Wirth J, Bureau S, Gunata Y and Razungles A, Biogeneration of $\mathrm{C} 13$ norisoprenoids compounds: experiments supportive for an apo-carotenoid pathway in grapevines. Anal Chem Acta 458:3-14 (2002).

11 Strauss CR, Wilson B, Anderson R and Williams PJ, Development of precursors of $\mathrm{C} 13$ nor-isoprenoid flavorants in Riesling grapes. Am J Enol Vitic 38:23-27 (1987). 
12 Park SK, Morrison JC, Adams DO and Noble AC, Distribution of free and glycosidically bound monoterpenes in the skin and mesocarp of Muscat of Alexandria grapes during development. F Agric Food Chem 39:514-518 (1991).

13 Castle R, http://www.brocku.ca/ccovi/res/reynolds/13.2aOevi_ 492a_Thesis_Final.pdf (2004). View in HTML. (Accessed January 2005).

14 Guedes de Pinho MP, Characterization de vins de la region des vinhos verde en Portugal. Diplome d'études approfondies en oenologie-ampelologie. Université de Bordeaux II (1991).

15 Rogerson FSS and Silva MCM, Investigations of classical monoterpene alcohol content in various single cultivar of Portuguese white wines, in 5th Symposium International d'oenologi, ed. by Lonvaud-Funel A. Lavoisier TEC_DOC, pp. 544-548 (1996).

16 Rogerson FSS, Studies on the application of enzymes in the production of wines from Portuguese grape varieties. $\mathrm{PhD}$ thesis, Universidade Católica, Porto, Portugal (1997).

17 Rogerson FSS, Grande HJ and Silva MCM, Free and enzyme enhanced monoterpenol content of Portuguese red wines from the Douro. Cienc Tecnol Aliment 2:169-173 (1999).

18 Carbonneau A, Observations sur vigne: codification des données agronomiques. Riv Vit Enol 4:37-45 (1991).
19 Di Stefano R, Proposition d'une méthode de préparation de l'échantillon pour la détermination des terpènes libres et glycosides des raisins et des vin. Bull OIV 64:219-223 (1991).

20 Di Stefano R and Maggiorotto G, Différences entre la composition terpèniques des cépages aromatiques, in Actes $d u$ Symposium International Connaissance aromatique des cépages et qualité des vin, Oenologie, Montpellier, pp. 107-112 (1993).

21 Di Stefano R, Borsa D, Maggiorotto G and Corino L, Trepeni e polifenoli di uve aromatiche a frutto colorato prodotte in piemonte. L'Enotecnico 4:75-85 (1995).

22 Di Stefano R, Bottero S, Pigella R, Borsa D, Bezzo G and Corino L, Precursori d' aroma glicosilati presenti nelle uve di alcune cultivar a frutto colorato. L'Enotecnico 3:63-74 (1998).

23 Pigella A, Bosso A, Di Stefano R, Corino L and Malerba G, Caratterizzazione varietale del Pinot nero attraverso lo studio dei polifenoli e dei precursori d'aroma. $R$ Vitic Enol 1:45-62 (1998).

24 Versini G, Rapp A, Reniero F and Mandery H, Structural identification and presence of some p-menth-1-endiols in grape products. Vitis 30:143-149 (1991). 